\title{
Orientational Order of a Lyotropic Chromonic Liquid Crystal by Polarized Raman Spectroscopy
}

Xuxia Yao, ${ }^{\dagger}$ Karthik Nayani, ${ }^{\dagger}$ Jung Ok Park, ${ }^{\dagger, \xi}$ and Mohan Srinivasarao ${ }^{*},+, \xi$

${ }^{\dagger}$ School of Materials Science and Engineering, Georgia Institute of Technology, Atlanta, Georgia 30332, USA

${ }^{\ddagger}$ School of Chemistry and Biochemistry, Georgia Institute of Technology, Atlanta, Georgia 30332, USA

${ }^{\S}$ Center for Advanced Research on Optical Microscopy, Georgia Institute of Technology, Atlanta, Georgia 30332, USA

\section{Corresponding Author}

*Email: Mohan@mse.gatech.edu; telephone: 404-894-9348 


\section{Supporting information}

S1. Phase diagram of Sunset Yellow FCF in water

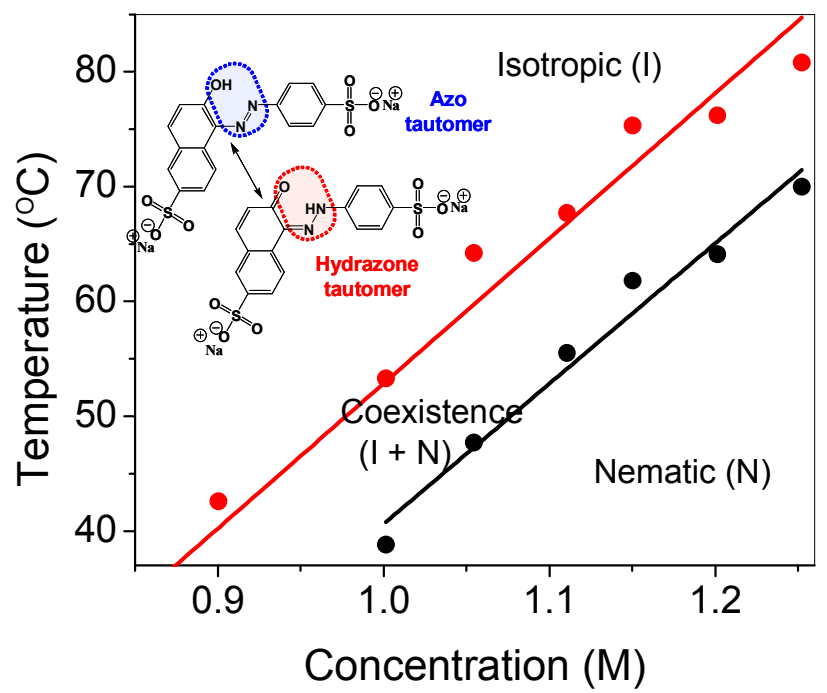

Figure S1. Phase diagram of Sunset Yellow FCF aqueous solution. Heating rate $0.4^{\circ} \mathrm{C} / \mathrm{min}$.

S2. Sample preparation

SSY was purchased from Sigma-Aldrich and purified by twice-precipitation in ethanol, filtration and 48-hour drying in the vacuum oven at room temperature.

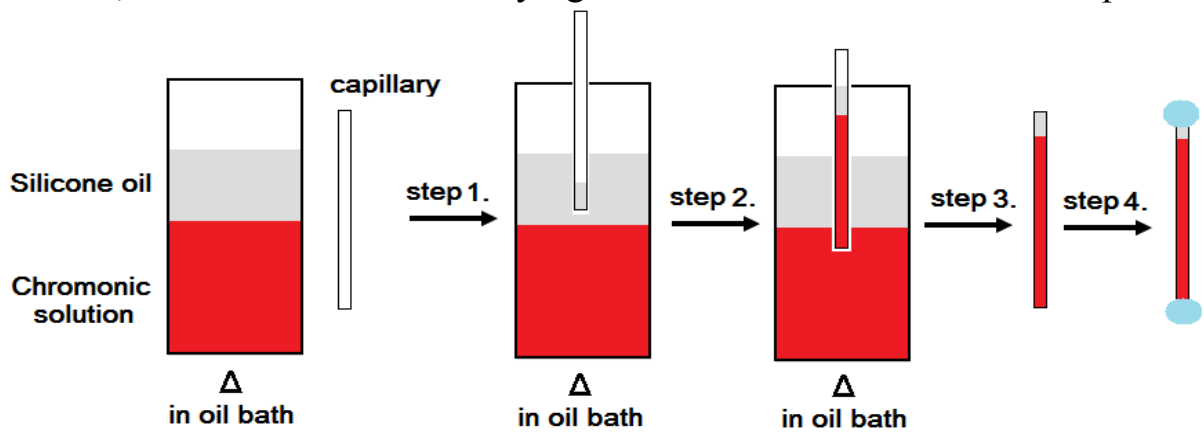

Step1: Heat the N phase of SSY solution to above the isotropic temperature.

Steps 2,3: Insert the flat capillary into the solution.

Step 4: Seal the two ends of the capillary by Permaoxy fast cure epoxy. 
S3. Experimental setup

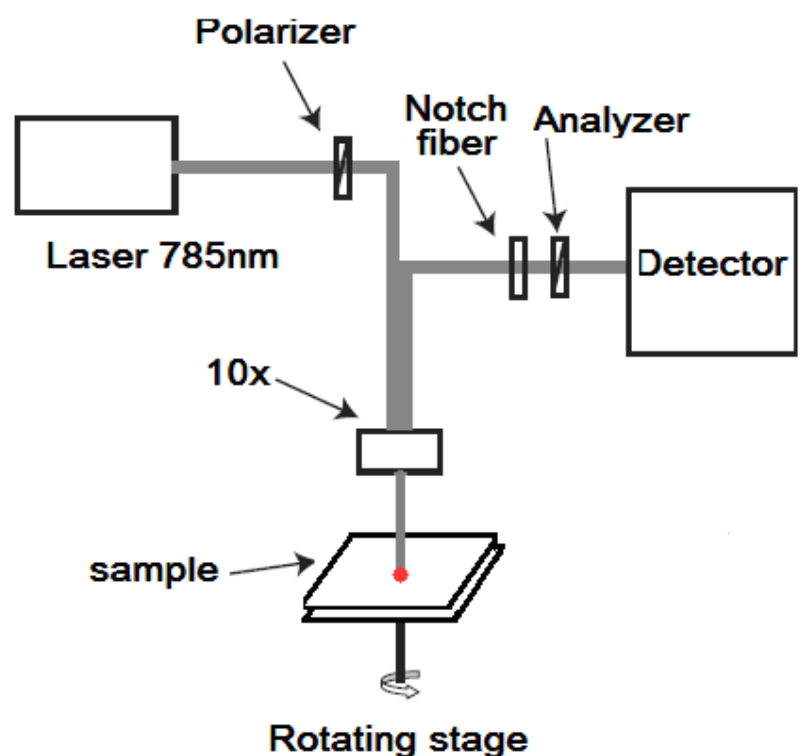

Figure S3. Schematic of the back-scattering geometry of a Kaiser 5000 Raman microscope. It is equipped with a $785 \mathrm{~nm}$ diode laser operating at $192 \mathrm{~mW}$ with a $10 \mathrm{x}$ microscope objective attached to the fiber optic probe head. Spectra were collected by the HoloGRAMNS software coupled to a charge coupled detector (CCD). Exposure time was set to 10 seconds and accumulations were set to 8 . Incident polarization was fixed, while the analyzer and sample stage were rotatable. 
S4. Raman spectra of SSY solutions in different phases.

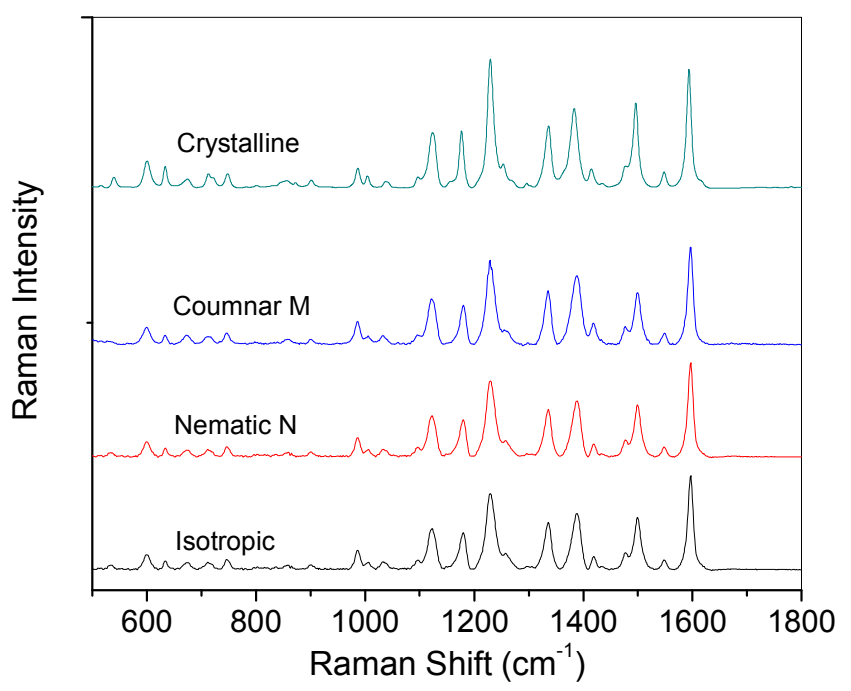

Figure S4. Raman spectra of SSY solutions in different phases. Isotropic phase with $0.7 \mathrm{M}$ concentration, nematic $\mathrm{N}$ phase with $1.1 \mathrm{M}$ concentration, columnar $\mathrm{M}$ phase with $1.7 \mathrm{M}$ concentration at room temperature, and crystalline solid, under parallel polarization.

S5. The bandwidth of phenyl C-C stretching $\left(1596 \mathrm{~cm}^{-1}\right)$ as function of temperature

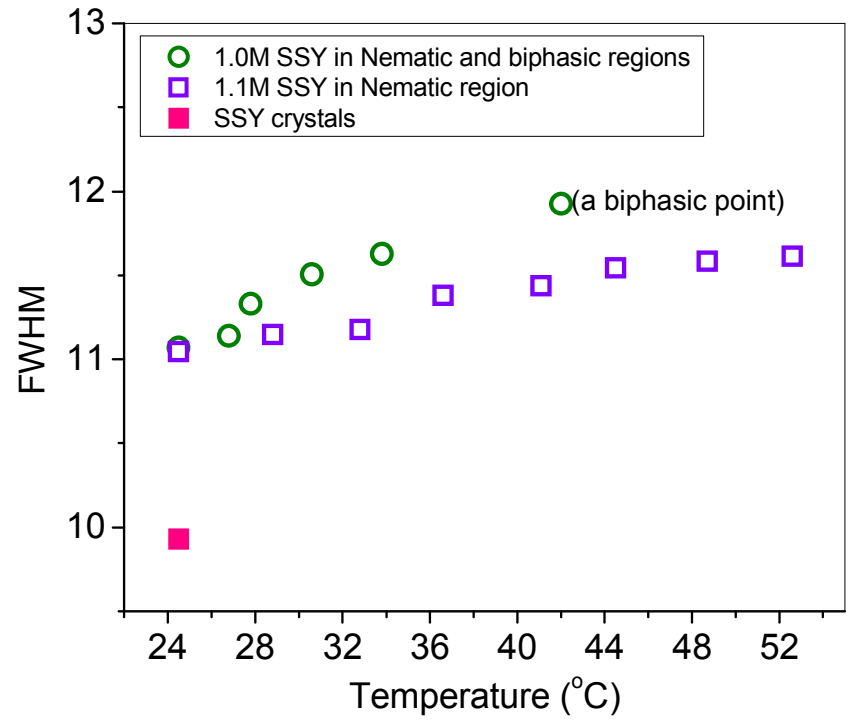


Figure S5. The Raman bandwidth of phenyl C-C stretching $\left(1596 \mathrm{~cm}^{-1}\right)$ as function of temperature. The green circle is for 1.0M SSY in the nematic phase, except one point in the biphasic region; the purple diamond is for 1.1M SSY in the nematic phase; the pink square is for SSY crystalline solid at room temperature. Those points are average values based on 20 spectra.

\section{S6. Raman spectrum of Orange II.}

Table S6: Experimental Infrared and Raman band position of Orange II with assignments to the calculated vibrational modes of 3; experimental Raman data of Sunset Yellow FCF.

\begin{tabular}{|c|c|c|c|c|c|c|c|c|c|c|c|c|c|}
\hline \multicolumn{8}{|c|}{ experimental: Orange II } & \multicolumn{4}{|r|}{ calculated: 3} & \multicolumn{2}{|c|}{ experimental: Sunset Yellow FCF } \\
\hline \multirow[b]{2}{*}{$\mathrm{IR}$} & & \multicolumn{6}{|c|}{$\operatorname{Raman}^{b}$} & & \multicolumn{2}{|c|}{ intensity $^{c}$} & \multirow[b]{2}{*}{ description $^{d}$} & \multicolumn{2}{|c|}{ Raman } \\
\hline & & \multicolumn{2}{|l|}{647.1} & \multicolumn{2}{|l|}{568.2} & \multicolumn{2}{|l|}{406.7} & & IR & $R$ & & \multicolumn{2}{|l|}{785} \\
\hline 1620 & $\mathrm{~s}$ & 1622 & $\mathrm{w}$ & & & & & 1626 & 13 & 1 & $v(\mathrm{CO}), \delta(\mathrm{NH}), \delta(\mathrm{naph})$ & & \\
\hline 1597 & $\mathrm{~m}$ & 1598 & vs & 1600 & vs & 1597 & vs & 1592 & 11 & 100 & $v(\mathrm{Ph}) \mathrm{8a}, \delta(\mathrm{Ph}) 9 \mathrm{a}, \delta(\mathrm{NH})$ & 1596 & vs \\
\hline 1584 & $\mathrm{w}$ & & & & & & & 1584 & 4 & 6 & $\delta(\mathrm{NH}), v(\mathrm{CO}), \delta(\mathrm{naph})$ & & \\
\hline 1567 & $\mathrm{~m}$ & & & & & & & 1563 & 9 & 0 & $v(\mathrm{Ph}) 8 \mathrm{~b}, \delta(\mathrm{Ph}) 3, \delta(\mathrm{NH}), v(\mathrm{NN}), \delta(\mathrm{naph})$ & & \\
\hline 1552 & $\mathrm{~m}$ & 1552 & $\mathrm{w}$ & 1554 & $\mathrm{w}$ & 1548 & vs & 1543 & 11 & 3 & $\delta($ naph), $\delta(\mathrm{NH})$ & 1546 & w \\
\hline \multirow[t]{2}{*}{1507} & vs & 1510 & $\operatorname{sh}$ & & & & & 1526 & 100 & 14 & $\delta(\mathrm{NH}), \delta(\mathrm{naph}), \nu(\mathrm{CN})$ & & \\
\hline & & 1499 & $\mathrm{~s}$ & 1499 & s & 1499 & w & 1489 & 2 & 20 & $v(\mathrm{Ph}) 19 \mathrm{a}, \delta(\mathrm{Ph}) 18 \mathrm{a}, v(\mathrm{CN})$ & 1496 & $\mathbf{s}$ \\
\hline 1483 & sh & 1481 & $\mathrm{w}$ & 1481 & $\mathrm{~m}$ & 1481 & s & 1470 & 4 & 3 & $\delta($ naph),$v(\mathrm{NN})$ & 1478 & w \\
\hline 1451 & $\mathrm{~m}$ & 1451 & w & 1452 & $\mathrm{w}$ & 1451 & $\mathrm{~m}$ & 1440 & 3 & 4 & $\delta$ (naph) & 1434 & w \\
\hline 1418 & $\mathrm{w}$ & 1416 & $\mathrm{~m}$ & 1417 & $\mathrm{~m}$ & 1416 & $\mathrm{~m}$ & 1417 & 9 & 23 & $v(\mathrm{Ph}) 19 \mathrm{~b}, \delta(\mathrm{Ph}) 1 \mathrm{sb}, v(\mathrm{CN}), \delta(\mathrm{NH}), \delta(\mathrm{naph})$ & 1419 & w \\
\hline 1403 & $\mathrm{w}$ & & & & & & & & & & & & \\
\hline 1391 & $\mathrm{w}$ & 1385 & s & 1386 & vs & 1386 & $\mathrm{~s}$ & 1397 & 9 & 22 & $v(\mathrm{Ph}) 19 \mathrm{~b}, \delta(\mathrm{Ph}) 18 \mathrm{~b}, \delta(\mathrm{NH}), v(\mathrm{CN})$ & 1390 & $\mathbf{s}$ \\
\hline \multirow{2}{*}{1335} & $\mathrm{w}$ & & & & & & & 1319 & 9 & 3 & $\delta($ naph $), \delta(\mathrm{Ph}), v(\mathrm{NN})$ & & \\
\hline & & 1334 & $\mathrm{~m}$ & 1334 & s & & & 1316 & 1 & 17 & $v(\mathrm{Ph}) 14, \delta(\mathrm{Ph}) 3, \delta(\mathrm{naph}), \delta(\mathrm{NH}), v(\mathrm{NN})$ & 1337 & m \\
\hline 1319 & $\mathrm{w}$ & & & & & & & 1301 & 1 & 2 & $v(\mathrm{Ph}) 14, \delta(\mathrm{Ph}) 3, \delta(\mathrm{naph}), v(\mathrm{NN}), \delta(\mathrm{NH})$ & & \\
\hline 1302 & w & & & 1302 & $\mathrm{w}$ & & & 1292 & 2 & 4 & $v(\mathrm{Ph}) 8 \mathrm{~b}, \delta(\mathrm{Ph}) 3, \delta(\mathrm{naph})$ & & \\
\hline 1268 & sh & & & & & & & & & & & & \\
\hline \multirow{2}{*}{1256} & $\mathrm{~s}$ & 1258 & $\mathrm{~m}$ & 1258 & $\mathrm{~m}$ & & & 1275 & 54 & 6 & $v(\mathrm{NN}), \delta(\mathrm{NH}), \delta(\mathrm{naph}), \delta(\mathrm{Ph}) 9 \mathrm{a}$ & 1258 & m \\
\hline & & 1250 & $\mathrm{~m}$ & & & & & 1236 & 14 & 7 & $\delta$ (naph) & & \\
\hline 1226 & vs & 1233 & s & 1230 & s & 1230 & $\mathrm{w}$ & 1230 & 11 & 15 & $v(\mathrm{Ph}), \delta(\mathrm{Ph}) 18 \mathrm{a}, \delta(\mathrm{naph}), v(\mathrm{CN}), \delta(\mathrm{NH})$ & 1229 & $\mathbf{s}$ \\
\hline 1210 & vs & 1210 & w & & & & & & & & & & \\
\hline $\begin{array}{l}1202 \\
1186\end{array}$ & $\begin{array}{l}\text { sh } \\
\text { vs }\end{array}$ & 1185 & 5 & 1184 & 5 & & & & & Oran & II: & 1180 & $\mathrm{~m}$ \\
\hline 1153 & $\mathrm{~m}$ & & & 1155 & w & 1155 & $\mathrm{w}$ & & & $\mathrm{R}$ & ${ }_{3} \mathrm{Na}$ & & \\
\hline 1121 & vs & 1123 & $\mathrm{~m}$ & 1121 & $\begin{array}{l}\mathrm{w} \\
\mathrm{w}\end{array}$ & & & & & & & 1122 & $\mathrm{~m}$ \\
\hline 1106 & $\mathrm{sh}$ & 1102 & $\mathrm{~m}$ & 1102 & $\mathrm{~m}$ & & & & & & & 1100 & w \\
\hline 1036 & vs & 1034 & $\mathrm{w}$ & & & & & & & & & 1034 & w \\
\hline 1006 & $\mathrm{~s}$ & 1009 & w & & & & & & & & & 1007 & w \\
\hline 986 & $\mathrm{~m}$ & 991 & $\mathrm{~m}$ & & & & & & & & & 984 & $\mathrm{~m}$ \\
\hline 952 & w & & & & & & & & & & & & \\
\hline & & & & & & & & & & - & 1-azo: $\mathrm{R}=\mathrm{H}$ & & \\
\hline $\begin{array}{l}\text { Data o } \\
\text { Data o }\end{array}$ & $\begin{array}{l}\text { Oran } \\
\text { Suns }\end{array}$ & $\begin{array}{l}\text { e II: J. } \\
\text { t Yello }\end{array}$ & $\begin{array}{l}\text { nys. } \\
\text { FCF }\end{array}$ & em. A & 05 & 2 & & & $\mathrm{R}=$ & ${ }_{3}^{3} \mathrm{H}$ & 3-azo: $\mathrm{R}=\mathrm{SO}_{3} \mathrm{H}$ & & \\
\hline
\end{tabular}

S7. Derivation of depolarization ratio.

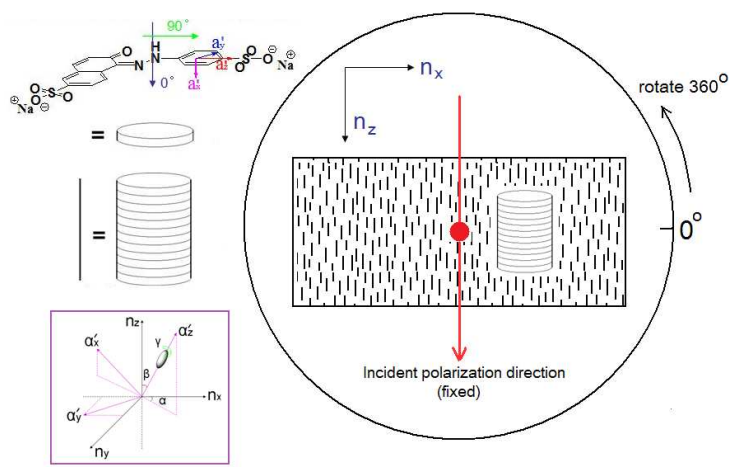


Figure S6. The directions of incident polarization, stacking columns and the Raman tensor of the stretching mode of the phenyl ring when the flat capillary is rotated. The $\alpha_{x}^{\prime}, \alpha_{y}^{\prime}$, and $\alpha_{z}^{\prime}$ are three components of the Raman tensor. The direction of $\alpha_{z}$ is the direction which the phenyl ring stretching vibration is strongly polarized along. $\mathrm{n}_{\mathrm{x}}, \mathrm{n}_{\mathrm{y}}, \mathrm{n}_{\mathrm{z}}$ are the reference frame. The red spot is position for the laser.

$$
I_{j i}(\theta)=I_{0} \iint_{\alpha} \int_{\gamma} f(\alpha, \beta, \gamma)\left(E_{j i}(\alpha, \beta, \gamma)\right)^{2} d \alpha d \beta d \gamma
$$

The above expression is for electric field equations for a homogeneous planar-aligned molecule with rotational degrees of freedom, $\alpha, \beta, \gamma$ and incident polarization direction, $\theta$, which is the angle between the long axis of the aggregate and incident polarization direction. $E_{/ /}$is used when the analyzer is parallel to the polarizer; $E_{\perp}$ is used when the analyzer is perpendicular to the polarizer.

$$
\begin{aligned}
& E_{/ / z x} \\
& =\alpha_{z}^{\prime}[\cos (\beta) \cos (\theta)-\sin (\beta) \sin (\theta) \cos (\alpha)]^{2} \\
& +\alpha_{x}^{\prime}[\sin (\beta) \cos (\theta)+\cos (\beta) \sin (\theta) \cos (\alpha)]^{2} \\
& +\alpha_{y}^{\prime}[\sin (\alpha) \sin (\theta)]^{2} \\
& E_{\perp z x} \\
& =\alpha_{z}^{\prime}[\cos (\beta) \cos (\theta)-\sin (\beta) \sin (\theta) \cos (\alpha)][\cos (\beta) \sin (\theta)+\sin (\beta) \cos (\theta) \cos (\alpha)] \\
& +\alpha_{x}^{\prime}[\sin (\beta) \cos (\theta)+\cos (\beta) \sin (\theta) \cos (\alpha)][\sin (\beta) \sin (\theta)-\cos (\beta) \cos (\theta) \cos (\alpha)] \\
& +\alpha_{y}^{\prime}[\sin (\alpha) \sin (\theta)][\sin (\alpha) \cos (\theta)]
\end{aligned}
$$

The orientational distribution function (ODF) can be approximated by expressing the exact ODF as a Fourier transforms in terms of the Wigner rotation matrices,

$D_{m n}^{L}(\alpha, \beta, \gamma)$

$f(\alpha, \beta, \gamma)=\sum_{L=0}^{\infty} \sum_{m=-L}^{+L} \sum_{n=-L}^{+L} \frac{2 L+1}{8 \pi^{2}}<D_{m n}^{L^{*}}>D_{m n}^{L}(\alpha, \beta, \gamma)$

$D_{m n}^{L}(\alpha, \beta, \gamma)=e^{-i m \alpha} d_{m n}^{L}(\beta) e^{-i n \gamma}$

Assumption and simplification of equations for the homogeneous planar-aligned unaxial sample:

1. We are unable to distinguish between any $180^{\circ}$ rotation due to the symmetry of the system, hence $\mathrm{L}, \mathrm{m}, \mathrm{n}$ must take even values.

2. In general the measured Raman bands are uniaxially symmetric $\left(\alpha_{z}^{\prime}>\alpha_{x}^{\prime}\left(=\alpha_{y}^{\prime}\right)\right)$, so all the order parameter associated with rotation about $\gamma$ are zero and only terms with $\mathrm{n}=0$ are non-zero.

3. Provided that the sample has mirror symmetry in the $x z, y z$ and xy planes, the imaginary components are eliminated.

4. Conventionally all the biaxial terms are set to zero for uniaxial systems, therefore only the order parameters $<\mathrm{P}_{200}>$ and $<\mathrm{P}_{400}>$ remain in the ODF. 


$$
\begin{aligned}
& f(\beta)=\frac{1}{8 \pi^{2}}\left[1+\frac{5}{2}<P_{200}>\left(3 \cos ^{2}(\beta)-1\right)+\frac{9}{8}<P_{400}>\left(3-30 \cos ^{2}(\beta)+35 \cos ^{4}(\beta)\right)\right] \\
& I_{\perp}(\theta)=I_{0} \int_{\alpha, \beta, \gamma} f(\beta)\left(E_{\perp}(\alpha, \beta, \gamma, \theta)\right)^{2} d \beta \\
& I_{/ /}(\theta)=I_{0} \int_{\alpha, \beta, \gamma} f(\beta)\left(E_{/ /}(\alpha, \beta, \gamma, \theta)\right)^{2} d \beta \\
& E_{/ / z x} \\
& =\alpha_{z}^{\prime}[\cos (\beta) \cos (\theta)-\sin (\beta) \sin (\theta)]^{2} \\
& +\alpha_{x}^{\prime}[\sin (\beta) \cos (\theta)+\cos (\beta) \sin (\theta)]^{2} \\
& E_{\perp z x} \\
& =\alpha_{z}^{\prime}[\cos (\beta) \cos (\theta)-\sin (\beta) \sin (\theta)][\cos (\beta) \sin (\theta)+\sin (\beta) \cos (\theta)] \\
& +\alpha_{x}^{\prime}[\sin (\beta) \cos (\theta)+\cos (\beta) \sin (\theta)][\sin (\beta) \sin (\theta)-\cos (\beta) \cos (\theta)]
\end{aligned}
$$

As a result, the depolarization ratio for all incident linear polarizations for a homogeneous planar-aligned liquid crystal cell in the backscattering geometry,

$$
\begin{aligned}
& R(\theta)=\frac{I_{\perp}(\theta)}{I_{/ /}(\theta)} \\
& R(\theta)=\frac{I_{\perp}(\theta)}{I_{/ /}(\theta)}=\frac{I_{0} \int_{\alpha, \beta, \gamma} f(\beta)\left(E_{\perp}(\alpha, \beta, \gamma, \theta)\right)^{2} d \beta}{I_{0} \int_{\alpha, \beta, \gamma} f(\beta)\left(E_{/ /}(\alpha, \beta, \gamma, \theta)\right)^{2} d \beta} \\
& =\frac{\int_{\beta}\left\{\frac{1}{8 \pi^{2}}\left[1+\frac{5}{2}<P_{200}>\left(3 \cos ^{2}(\beta)-1\right)+\frac{9}{8}<P_{400}>\left(3-30 \cos ^{2}(\beta)+35 \cos ^{4}(\beta)\right)\right]\right\}}{\int_{\beta}\left\{\frac{1}{8 \pi^{2}}\left[1+\frac{5}{2}<P_{200}>\left(3 \cos ^{2}(\beta)-1\right)+\frac{9}{8}<P_{400}>\left(3-30 \cos ^{2}(\beta)+35 \cos ^{4}(\beta)\right)\right]\right\}} \\
& \frac{\left\{\alpha_{z}^{\prime}[\cos (\beta) \cos (\theta)-\sin (\beta) \sin (\theta)][\cos (\beta) \sin (\theta)+\sin (\beta) \cos (\theta)]\right.}{\left\{\alpha_{z}^{\prime}[\cos (\beta) \cos (\theta)-\sin (\beta) \sin (\theta)]^{2}\right.} \\
& \left.+\alpha_{x}^{\prime}[\sin (\beta) \cos (\theta)+\cos (\beta) \sin (\theta)][\sin (\beta) \sin (\theta)-\cos (\beta) \cos (\theta)]\right\}^{2} d \beta \\
& =\frac{\left.(-1+r)^{2}[\sin (\beta) \cos (\theta)+\cos (\beta) \sin (\theta)]^{2}\right\}^{2} d \beta}{-56\left(3+4 r+8 r^{2}\right)+40<P_{200}>(-1+r)(3+4 r)(1+3 \cos (2 \theta))} \\
& \quad+105<P_{400}>\cos (4 \theta)
\end{aligned}
$$


S8. Conoscopic Images

Conoscopic interference Figures for SSY chromonics in a rectangular capillary 50x500um, using $633 \mathrm{~nm}$ light source
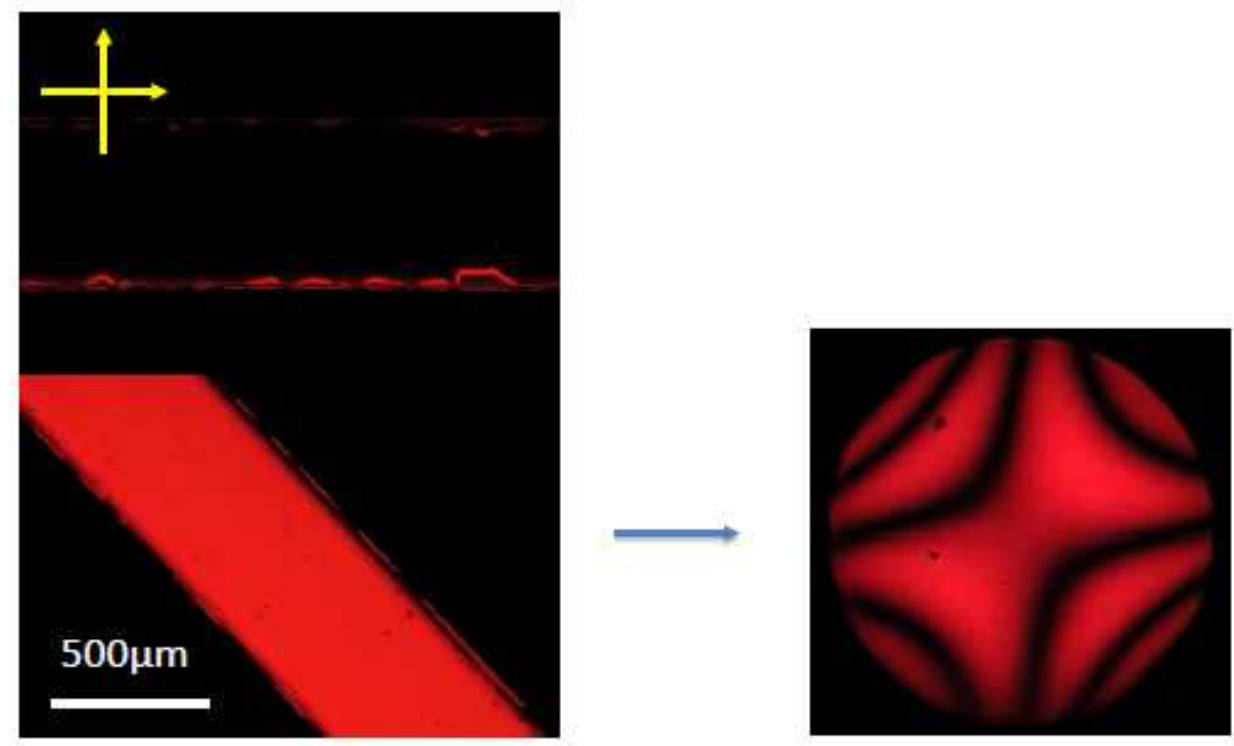

Objective $63 \times \mathrm{NA}=0.8$ 